\title{
Valor do Dilatador Higroscópico para Visualização do Canal Endocervical na Conização com Cirurgia de Alta Frequêencia
}

\author{
Value of the Hygroscopic Dilator for Visualization of the Endocervical \\ Canal in Conization by Loop Electrosurgical Excision Procedure \\ Maria Silvana Cardoso Ferreira ${ }^{1}$, Julisa Chamorro Lascasas Ribalta ${ }^{2}$, \\ José Focchi², Saadi Abrahão Taha², João Norberto Stávale ${ }^{2}$, \\ Elizabete Rautman Cezarino Linhares ${ }^{2}$, Edmund Chada Baracat ${ }^{2}$
}

\section{RESUMO}

Objetivo: avaliar a eficácia do dilatador higroscópico para auxiliar o exame colposcópico do canal endocervical, em pacientes com lesão de alto grau e colposcopia insatisfatória.

Métodos: estudo prospectivo no qual foram incluidas 62 pacientes com exames colposcópicos insatisfatórios e citologia sugestiva de lesão de alto grau. Todas foram submetidas à dilatação do canal por meio de dilatador higroscópico. Após a dilatação os novos achados colposcópicos foram registrados e procedeu-se à conização por cirurgia de alta freqüencia (CAF). Comparamos a incidência de comprometimento neoplásico das margens cirúrgicas operatórias entre as pacientes que modificaram o exame para satisfatório e aquelas que persistiram insatisfatórios. Para comparação entre a incidência de margens comprometidas e a incidência de doença residual, foram utilizados dois grupos controle retrospectivos: grupo GinSat (n=35): pacientes com colposcopias insatisfatórias; grupo GSat $(n=38)$ : pacientes com colposcopias satisfatórias e atipia de localização endocervical.

Resultados: em 80,6\% dos casos a visão colposcópica após a dilatação foi satisfatória. Destes, 80,4\% apresentaram margens livres de doença. A incidência de margens livres nas pacientes com colposcopias insatisfatórias persistentes após a dilatação foi de 36,3\%. O comprometimento das margens cirúrgicas ocorreu em 28,0\% no grupo submetido à dilatação, em 28,5\% dos casos no grupo GinSat e em 31,5\% no grupo GSat No acompanhamento verificou-se a incidência de 7,5\% de doença residual nas pacientes submetidas à dilatação. No grupo GinSat a incidência de doença residual foi de 28,6\% e no grupo GSat foi de 28,9\%.

Conclusão: o uso do dilatador higroscópico facilitou a visualização de lesões de difícil acesso ao exame colposcópico, permitindo diminuir a porcentagem de doença residual em pacientes com colposcopias insatisfatórias submetidas à conização por cirurgia de alta freqüência.

PALAVRAS-CHAVE: Colposcopia. Colposcopia insatisfatória. Colo do útero: lesões préneoplásicas. Conização.

${ }^{1}$ Disciplina de Tocoginecologia da Faculdade de Medicina de Itajubá - FMIT

${ }^{2}$ Disciplina de Ginecologia, Departamento de Ginecologia, Universidade Federal de São Paulo - UNIFESP

Correspondência:

Maria Silvana Cardoso Ferreira

Rua Cel. Francisco Braz 368 - Centro

37500-000 - Itajubá - MG

Fone: (35) 3623-6193

\section{Introdução}

Em paciente portadora de exame citopatológico cervicovaginal anormal, a avaliação colposcópica é procedimento necessário, conduzindo ao diagnóstico correto e escolha de opção terapêutica, por localizar e avaliar a extensão da lesão já suspeitada ${ }^{1}$. 
O exame colposcópico é considerado satisfatório quando toda a extensão da junção escamocolunar (JEC) e os limites craniais das lesões são adequadamente visualizados. A freqüência de colposcopias insatisfatórias varia de 4 a 53\% dos exames, sendo influenciada pela experiência do colposcopista, idade da paciente e a idade de instalação da menopausa ${ }^{2}$.

As lesões intra-epiteliais cervicais são na maioria das vezes colposcopicamente visíveis em toda sua extensão, especialmente em pacientes jovens. A proporção de lesões localizadas no canal endocervical aumenta com a idade, uma vez que a JEC e a zona de transformação movem-se em direção ao interior do canal no climatério ${ }^{3}$. Por outro lado, paciente idosa com exame citopatológico anormal tem maior chance de apresentar câncer invasor do que a jovem ${ }^{4,5}$. Com isso, a ansiedade provocada por esfregaço colpocitológico anormal alia-se à incerteza de uma colposcopia inadequada.

Muitas técnicas têm sido desenvolvidas para melhor avaliação colposcópica do canal endocervical uterino. Muitas vezes uma leve pressão afastando para trás o lábio anterior ou o posterior, utilizando um bastonete envolto em algodão, estende o campo colposcópico o suficiente para se completar a avaliação ${ }^{6}$. Para visualização mais ampla do canal, empregam-se afastadores especiais do tipo Hillemann ou tipo Mencken-KoganSchiwartche. De acordo com Prendeville et al. ${ }^{7}$, a estrogenioterapia por via oral em mulheres menopausadas, com citologia anormal e colposcopia insatisfatória, apresenta taxa de sucesso de $64 \%$ dos casos na visualização da zona de transformação.

É possivel ainda avaliar a mucosa endocervical por meio do microcolpo-histeroscópio. Este instrumento permite observar diretamente a mucosa endocervical com aumentos de 1:20, 1:60 e $1: 150^{8}$. Outra técnica freqüentemente utilizada em pacientes com colposcopia insatisfatória é a curetagem endocervical.

No entanto, todas as técnicas descritas têm vantagens e desvantagens. Os afastadores endocervicais são, com freqüência, mal tolerados, podendo induzir sangramento, que dificulta a visão colposcópica. A curetagem endocervical é dolorosa e não localiza a área atípica. Andersen et al. ${ }^{9}$ relatam em seu estudo que a sensibilidade do procedimento é de 55\% quando comparada à escova endocervical, que alcança índices de até $92 \%$ de acertos diagnósticos. Poucos trabalhos na literatura avaliam a estrogenioterapia em pacientes com colposcopia insatisfatória. Os trabalhos encontrados são conflitantes em relação à dose uti- lizada e efeitos colaterais, além de apresentarem número pequeno de $\operatorname{casos}^{7,10}$. Por sua vez, a microcolpo-histeroscopia requer tempo de execução mais prolongado, o custo do instrumental é alto e exige pessoal habilitado, restringindo seu uso às instituições especializadas.

Finalmente, a conização diagnóstica é considerada, por muitos autores, o melhor procedimento diagnóstico e terapêutico nos casos de neoplasia intra-epitelial de alto grau que se estenda para o canal ${ }^{10,11}$. Dentre as técnicas cirúrgicas empregadas, a conização utilizando cirurgia de alta freqüência $(\mathrm{CAF})$ permite o bom estudo histopatológico da peça, além de seu baixo custo e menores índices de complicações.

Recentes estudos têm analisado o uso de dilatadores higroscópicos do canal endocervical. Os mais usados são: Lamicel ${ }^{\circledR}$, formado por uma esponja absorvente de polivinil, impregnada com 500 mg de sulfato de magnésio, e o Dilapan ${ }^{\circledR}$, composto por Aquacryl ${ }^{\mathbb{}}$, um hidrogel de base acrílica ${ }^{12-15}$.

O propósito deste estudo foi verificar se a aplicação do dilatador promoveria visibilidade colposcópica necessária para dirigir a exérese por CAF, melhorando os resultados terapêuticos em pacientes com exame citopatológico compatível com lesão de alto grau e colposcopia insatisfatória.

\section{Pacientes e Métodos}

Foram incluídas e avaliadas prospectivamente 62 pacientes com citologia sugestiva de neoplasia intra-epitelial de alto grau e exame colposcópico insatisfatório. O estudo foi desenvolvido no período de novembro de 1999 a agosto de 2002 na Universidade Federal de São Paulo UNIFESP, Faculdade de Medicina de Itajubá e Instituto Brasileiro de Controle do Câncer.

Obedecidos os critérios de inclusão e após assinarem o termo de consentimento livre e informado, as pacientes foram encaminhadas para a aplicação do dilatador. Em posição ginecológica fez-se a introdução de espéculo de Collins para observação da cérvice. Após a limpeza com soro fisiológico observaram-se, pela colposcopia, o colo e as paredes vaginais. A seguir, fez-se a aplicação do ácido acético a 3\% e inspeção da ectocérvice, do canal endocervical e das paredes vaginais.

Para inserção do dilatador higroscópico procedeu-se de maneira suave, sem ultrapassar o orificio interno do colo, alcançando até $1,5 \mathrm{~cm}$ na endocérvice. A profundidade alcançada foi medida no dilatador utilizando-se um paquímetro. Feita a inserção do dilatador, o espéculo vaginal foi reti- 
rado e a paciente foi orientada para permanecer na sala de espera, por periodo de três horas. Para a retirada do dilatador as pacientes foram submetidas a novo exame especular e o dilatador foi levemente tracionado pelo fio guia, localizado em sua parte distal.

Nova colposcopia foi realizada, com aplicação de solução fisiológica para limpeza e observação com filtro verde. A seguir foi aplicada solução de ácido acético a 3\% para observação da endocévice após dilatação. Definida a localização da lesão e da JEC procedeu-se à excisão com alça de alta freqüência. As alças mais utilizadas foram a de $1,5 \mathrm{~cm}$ e $1,7 \mathrm{~cm}$ de comprimento, ambas com $1 \mathrm{~cm}$ de largura.

A peça cirúrgica foi aberta e fixada em placa de isopor e submersa em frasco contendo formol a $10 \%$.

O processamento histopatológico fez-se com cortes perpendiculares à JEC, em fragmentos de 2 a $3 \mathrm{~mm}$, incluindo todo material em bloco de parafina. Cada secção foi desidratada em álcool etílico, diafanizada em xilol e incluída em parafina à temperatura de $58^{\circ} \mathrm{C}$. De cada bloco fizeram-se cortes com micrótomo, obtendo de 4 a 5 lâminas, as quais foram coradas com hematoxilina-eosina. A leitura dos espécimes histopatológicos foi revista por um único patologista do Departamento de Patologia da UNIFESP para uniformização dos diagnósticos.

Fez-se o seguimento pós-cirúrgico com consultas aos 15 dias e 30 dias após a cirurgia e a cada três meses no primeiro ano, semestralmente no segundo e, a seguir, avaliação anual.

Definiu-se como doença residual o diagnóstico histopatológico de qualquer grau de neoplasia intra-epitelial no intervalo de até seis meses após o ato cirúrgico. Recidiva seria o surgimento de lesão histopatológica após seis meses ou dois exames citopatológicos trimestrais sem alterações.

Para a avaliação dos resultados utilizaramse como dados de comparação dois grupos controles retrospectivos. O grupo GinSat, composto por 35 pacientes com colposcopias insatisfatórias, e o grupo Gsat, com 38 pacientes com colposcopias satisfatórias, porém, com lesões que comprometiam a endocérvice. Todas as pacientes dos grupos controles foram submetidas à conização por CAF nos ambulatórios de Oncologia Clínica e Patologia do Trato Genital Inferior da UNIFESP - EPM, no período de 1993 a 1999.

O teste exato de Fisher foi utilizado para a comparação entre margens cirúrgicas, colposcopia satisfatória e colposcopia insatisfatória após dilatação, margens cirúrgicas no grupo estudado e no grupo GinSat, margens cirúrgicas no grupo estudado e no grupo GSat, doença residual no grupo estudado e no grupo GinSat, e doença residual e no grupo estudado e grupo GSat. Em todos os casos, o nível de rejeição para hipótese de nulidade foi fixado valor menor ou igual a 0,05 (5\%), sendo utilizado o software estatístico SPSS versão 11.5 para efetuar os cálculos da análise.

O estudo foi aprovado pelo Comitê de Ética em Pesquisa Médica da Universidade Federal de São Paulo, Faculdade de Medicina de Itajubá e Instituto Brasileiro de Controle de Câncer.

\section{Resultados}

Sessenta e duas pacientes participaram do estudo. Destas, 50 tiveram seus exames colposcópicos modificados para satisfatórios após a aplicação do dilatador. Dentre as 12 pacientes que persistiram com colposcopias insatisfatórias, em três foi possivel visualizar o aspecto colposcópico anormal endocervical, porém não a JEC.

Evidenciou-se localização da atipia exclusivamente endocervical em 44 casos. Nas restantes 18 pacientes, a lesão ocupava as proximidades do orificio externo, adentrando o canal.

O dilatador provoca além da expansão do canal, o amolecimento do colo, permitindo maior facilidade no uso dos afastadores endocervicais. Foi observado também maior freqüência de traumatismo com presença de áreas de destacamento mais evidentes de tecido após o uso do dilatador Dilapan ${ }^{\circledR}$. Houve dificuldade para a retirada deste dilatador na maioria dos casos. O dilatador Dilapan ${ }^{\circledR}$ adere fortemente aos tecidos vizinhos e sofre maior aumento de volume na sua porção distal. Este efeito levou a traumatismo na sua retirada. O mesmo não ocorreu com o dilatador Lamicel ${ }^{\circledR}$, tanto na inserção quanto na sua retirada.

Considerando-se os resultados anatomopatológicos do produto final da conização, foram observados três casos de carcinoma invasor. Em 12 pacientes não foram encontradas lesões residuais e oito casos apresentaram NIC I, sete NIC II e 32 NIC III.

Foram excluídas do estudo as três pacientes com carcinoma invasor e duas outras que apresentaram, na peça operatória, dano tecidual impossibilitando a avaliação anatomopatológica. Restaram 57 casos que compõem a casuística para a análise das demais variáveis do estudo.

Em 46 casos a colposcopia após o dilatador foi satisfatória. Destes, $37(80,4 \%)$ apresentaram margens de ressecção livres de doença. O mesmo ocorreu em quatro $(36,3 \%)$ do total de 11 pacien- 
tes que permaneceram com colposcopia insatisfatória após a dilatação. A comparação entre os resultados mostrou significância estatística (Tabela 1).

Tabela 1 - Comprometimento de margens cirúrgicas de ressecção de acordo com a avaliação colposcópica após a dilatação endocervical em 57 pacientes submetidas a conização por cirurgia de alta freqüência.

\begin{tabular}{lrrrrrrr}
\hline Margens cirúrgicas & \multicolumn{5}{c}{ Colposcopia após dilatação } \\
& Satisfatória & \multicolumn{1}{c}{ Insatisfatória } & \multicolumn{2}{c}{ Total } \\
& $\mathbf{n}$ & $\%$ & $\mathbf{n}$ & $\%$ & $\mathbf{n}$ & $\%$ \\
\hline Livres & 37 & 80,4 & 4 & 36,4 & 41 & 71,9 \\
Comprometidas & 9 & 19,6 & 7 & 63,6 & 16 & 28,1 \\
Total & 46 & 100,0 & 11 & 100,0 & 57 & 100,0
\end{tabular}

Teste exato de Fisher $(p=0,007)$

Ao analisarmos as margens cirúrgicas, verificamos que em 16 casos $(28,1 \%)$ do grupo submetido à dilatação, as margens cirúrgicas apresentaram-se comprometidas pela neoplasia; o mesmo ocorreu em 10 pacientes $(28,6 \%)$ do grupo GinSat $(p=1,00)$ e em $12(28,1 \%)$ do grupo GSat $(\mathrm{p}=0,819)$. Não houve, portanto, associação entre a aplicação do dilatador e comprometimento das margens cirúrgicas.

Das 57 pacientes, quatro foram excluídas por não terem completado seis meses de acompanhamento, restando 53 para as subseqüentes avaliações. O tempo médio de seguimento para todas as pacientes foi de 15,5 meses, sendo que $61,3 \%$ dos casos tinham mais de um ano de acompanhamento.

Observamos na Tabela 2 que, das 53 pacientes submetidas à dilatação, 49 (92,4\%) permaneceram livres de doença após seis meses; da mesma forma, 25 pacientes $(71,4 \%)$ do grupo GinSat não apresentaram doença residual. A análise estatística pelo teste exato de Fisher mostrou relação entre a ausência de doença residual e o uso do dilatador.

Tabela 2 - Doença residual no grupo de pacientes submetidas à dilatação comparado às 35 pacientes do grupo controle com colposcopia insatisfatória (GinSat).

\begin{tabular}{lcrrrrr}
\hline Diagnóstico & \multicolumn{2}{c}{ Grupo GinSat } & \multicolumn{2}{c}{ Dilatação } & \multicolumn{2}{c}{ Total } \\
& $\mathbf{n}$ & \multicolumn{1}{c}{$\%$} & \multicolumn{1}{c}{ n } & \multicolumn{1}{c}{$\%$} & \multicolumn{1}{c}{ n } & \multicolumn{1}{c}{$\%$} \\
\hline Livre de doença & 25 & 71,4 & 49 & 92,4 & 74 & 84,1 \\
Doença residual & 10 & 28,6 & 4 & 7,6 & 14 & 15,9 \\
Total & 35 & 100,0 & 53 & 100,0 & 88 & 100,0
\end{tabular}

Teste exato de Fisher $(p=0,015)$

Pela Tabela 3 evidenciamos que 27 (71,0\%) pacientes do grupo GSat permaneceram livres de doença. No grupo submetido à dilatação, 49 (92,4\%) permaneceram livres de doença residual. A análise dos dados mostrou relação entre a ausência de doença residual e a aplicação do dilatador.

Tabela 3 - Doença residual no grupo de pacientes submetidas à dilatação comparado às as 38 pacientes do grupo controle que apresentavam colpsocopia satisfatória (Gsat).

\begin{tabular}{lcrrrrr}
\hline Diagnóstico & \multicolumn{2}{c}{ GSat } & \multicolumn{2}{c}{ Dilatação } & \multicolumn{2}{c}{ Total } \\
& $\mathbf{n}$ & $\%$ & \multicolumn{1}{c}{$\mathbf{n}$} & \multicolumn{1}{c}{$\%$} & \multicolumn{1}{c}{ n } & $\%$ \\
\hline Livre de doença & 27 & 71,1 & 49 & 92,4 & 38 & 41,8 \\
Doença residual & 11 & 28,9 & 4 & 7,6 & 53 & 58,2 \\
Total & 38 & 100,0 & 53 & 100,0 & 91 & 100,0
\end{tabular}

Teste exato de Fisher $(p=0,01)$

De 12 pacientes que não apresentaram lesão na peça operatória da conização, seis apresentaram biópsias negativas após a dilatação e submeteram-se à conização por CAF por discordância cito-histopatológica. As seis pacientes restantes apresentaram, à biópsia, lesões de alto grau que provavelmente foram extirpadas pela pinça saca-bocado. Das oito pacientes que apresentaram NIC I no resultado final do produto da conização, sete também foram submetidas à conização por discordância entre a citologia e a histopatologia.

As pacientes que não apresentaram doença neoplásica no exame histopatológico da peça operatória foram acompanhadas com exames colpocitológicos e colposcópicos, por tempo médio de 16,4 meses. Nenhuma paciente desse grupo apresentou doença residual ou recidiva.

As pacientes que apresentaram persistência de doença residual foram submetidas a histerectomia ou nova CAF. Não foi observado nenhum caso de câncer invasor após o tratamento conservador. Uma paciente apresentou recidiva após 14 meses de tratamento e foi submetida a um novo procedimento de conização por CAF.

\section{Discussão}

O objetivo do tratamento das lesões intraepiteliais é a prevenção do câncer invasor. Embora o risco de portadoras de lesões precursoras submetidas a tratamento conservador desenvolver câncer invasor seja baixo, este ainda permanece quatro a cinco vezes mais freqüente do que na população em geral e não se reduz com o passar do tempo ${ }^{16}$.

$\mathrm{O}$ estabelecimento de fatores de risco que possam influenciar a persistência das lesões intraepiteliais, e o surgimento e a progressão de novas 
lesões para invasão, têm sido objetivo de muitas pesquisas ${ }^{17}$. O comprometimento das margens de ressecção cirúrgica, a idade da paciente, a extensão da área atípica, o comprometimento do canal endocervical, o envolvimento das criptas glandulares e a colposcopia insatisfatória são os fatores de risco mais estudados ${ }^{18,19}$.

Para a exérese completa da área com atipia, visando evitar a permanência de focos residuais, responsáveis por recorrências, é necessária a avaliação colposcópica minuciosa, além do estudo da localização da área alterada em relação ao canal endocervical. O estudo do canal endocervical de pacientes com colposcopia insatisfatória foi possível após o uso do dilatador endocervical em $80,6 \%$ dos casos. Esse resultado é concordante com a maioria dos autores, que mostram indices variando de 76 a $94 \%$ de sucesso ${ }^{12,13,15,20}$.

Neste estudo, a média de idade das pacientes, 46,2 anos, coincide com aquela referida na literatura, acentuando a relação entre o aumento da idade e a não-visualização da $\mathrm{JEC}^{5}$. O grupo GinSat apresentou média de idade de 47,8 anos e o grupo GSat, 42,7 anos.

A avaliação do canal após a dilatação exige cuidado na manipulação dos tecidos devido a maior fragilidade e destacamento das áreas atípicas que ocorreram após a dilatação. Outros fatores como os baixos niveis de estrogênio, a presença de focos de epitélio atípico isolados da lesão principal e longe da JEC, o forte pregueamento da mucosa endocervical formando relevo polipóide, bem como os processos inflamatórios, dificultaram a interpretação das imagens intra-cervicais, como já salientara Rieper ${ }^{21}$.

O estudo histopatológico dos espécimes de conização mostrou, em $32,2 \%$ das vezes, doença menos grave em um ou dois graus do que aquela diagnosticada pela citologia e/ou biópsia. Resultado semelhante é descrito por Lopes et al. ${ }^{22} \mathrm{em}$ pacientes com colposcopias insatisfatórias submetidas à conização a frio.

A presença de doença nas margens cirúrgicas de ressecção foi observada em percentual inferior no grupo de pacientes que apresentaram colposcopia satisfatória após o uso do dilatador endocervical, do que naquelas em que houve persistência de exame colposcópico insatisfatório após a dilatação. Podemos observar na literatura que pacientes com exames colposcópicos satisfatórios, apresentam melhores índices de ressecção completa das lesões ${ }^{23,24}$.

Na comparação entre os resultados do grupo estudado e do grupo GinSat, verificamos diminuição significante de doença residual no grupo com dilatação. O mesmo resultado foi observado quan- do comparamos o grupo submetido à dilatação e o grupo GSat. Entretanto, não houve diferença entre as porcentagens de comprometimento de margens entre as pacientes submetidas à dilatação e aquelas do grupo GinSat e do grupo GSat.

Acreditamos que a extensão e a altura das lesões no canal não permitiram, mesmo após a aplicação do dilatador, sua retirada com maior margem histopatológica. Observamos que nem sempre os limites colposcópicos das margens livres de lesão coincidiram com os limites histopatológicos. O único trabalho na literatura no qual se utilizaram dilatadores higroscópicos para auxiliar o tratamento de pacientes com colposcopia insatisfatória e lesão de alto grau foi realizado por Johnson e Brady ${ }^{14}$. Os autores tornaram a colposcopia satisfatória em 25 pacientes do total de 41 casos e em seguida procederam à cauterização das lesões. Após 5 anos de seguimento observaram $43 \%$ de recidiva.

Poucos trabalhos na literatura estudaram a eficácia do tratamento de pacientes com suspeita de lesão de alto grau e colposcopia insatisfatória. Oyesanya et al. ${ }^{11}$, comparando conização por CAF com conização a frio em pacientes com colposcopia insatisfatória, relatam $16,3 \%$ de comprometimento das margens cirúrgicas de ressecção e $7 \%$ de recidiva. Os autores realizaram os cones com alça de $2 \mathrm{~cm}$ de comprimento. As alças utilizadas neste estudo foram de $1,5 \mathrm{~cm}$ e $1,7 \mathrm{~cm}$ de comprimento, ambas com $1 \mathrm{~cm}$ de largura. Alças maiores foram evitadas para maior segurança do procedimento, que foi realizado em regime ambulatorial e com anestesia local.

Acreditamos também que em número considerável de pacientes, após a dilatação, foi alcançada maior quantidade de tecido anormal, tendo como conseqüência a destruição total das lesões. Esta distribuição pode ser devida tanto ao efeito térmico que se prolonga além da linha de corte, quanto pelos efeitos hemostático, imunológico e/ou inflamatório ${ }^{23}$.

O uso de meios que permitam a visualização colposcópica da região do colo de difícil acesso, como o canal endocervical, abre novo campo de estudo e conhecimento para imagens ainda pouco familiares ao exame colposcópico de rotina, como as que se localizam no terço médio e mais raramente no terço superior da endocérvice ${ }^{25}$.

Apesar do número pequeno de casos e da comparação com grupos controles retrospectivos, os dados sugerem que o uso dos dilatadores higroscópicos melhorou a visualização das lesões endocervicais, permitindo a diminuição da porcentagem de doença residual em pacientes com colposcopia insatisfatória. 
ABSTRACT

Objective: to evaluate the effectiveness of the hygroscopic dilator in the colposcopic examination of the endocervical canal in patients with high-grade lesion in the cytopathology and unsatisfactory colposcopy.

Methods: prospective study, including 62 patients with unsatisfactory colposcopic examination and cytology compatible with high-grade intraepithelial lesion. The patients were submitted to dilation of the endocervical canal by means of a hygroscopic dilator. After dilation, the new colposcopic findings were recorded, and then conization was made through loop electrosurgical excision procedure. The incidence of neoplasic involvement of surgical margins was compared between patients with examinations modified toward satisfactory results and those that remained with unsatisfactory colposcopy. In order to compare the incidence of involved margins and the incidence of residual disease, two retrospective control-groups were used: the GinSat group $(n=35)$ : patients with unsatisfactory colposcopy; GSat group $(n=38)$ : patients with satisfactory colposcopy and endocervical atypy.

Results: $80.6 \%$ of the cases presented satisfactory colposcopic vision after dilation. $80.4 \%$ of those presented disease-free resection margins. The incidence of disease-free resection margins in patients with persistent unsatisfactory colposcopy after dilation was $36.3 \%$. Affected surgical margins occurred in $28 \%$ of the group that had undergone dilation, $28.5 \%$ of the cases in GinSat group, and $31.5 \%$ in the Gsat group. Follow-up showed the incidence of residual disease in $7.5 \%$ of the patients under dilation, $28.5 \%$ in the GinSat group and $28.9 \%$ in the GSat group.

Conclusion: the use of hygroscopic dilation improved visualization of lesions of difficult access to the colposcopic examination, thus permitting reduction in the percentage of residual neoplasic disease in patients with unsatisfactory colposcopy treated with loop electrosurgical excision procedure.

KEY WORDS: Colposcopy. Unsatisfactory colposcopy. Cervical intraepithelial neoplasia.

\section{Referências}

1. Ministério da Saúde. Instituto Nacional do Câncer. Normas e recomendações. Recomendações básicas para o controle do câncer do colo útero no Brasil. Rev Bras Cancerol 2000; 46:23-33.

2. Rochelson B, Krumholz BA. The "unsatisfactory" colposcopic examination. J Reprod Med 1983; 28:131-6.

3. Boulanger JC, Gondry J, Verhoest P, Capsie C, Najas S. Treatment of CIN after menopause. Eur J Obstet Gynecol Reprod Biol 2001; 95:175-80.
4. Shingleton HM, Partridge EE, Austin JM. The significance of age in the colposcopic evaluation of women with atypical Papanicolaou smears. Obstet Gynecol 1977; 49:61-4.

5. Wetrich DW. An analysis of the factors involved in the colposcopic evaluation of 2194 patients with abnormal Papanicolaou smears. Am J Obstet Gynecol 1986; 154:1339-49.

6. Singer A, Monaghan JM, Quek SC, Deery ARS. Colposcopia: patologia e tratamento do trato genital inferior. $2^{\mathrm{a}}$ ed. Rio de Janeiro: Revinter; 2002. p.123-4.

7. Prendeville WJ, Davies WA, Davies JO, Shepherd AM. Medical dilatation of the non-pregnant cervix: the effect of ethinyl oestradiol on the visibility of the transformation zone. Br J Obstet Gynaecol 1986; 93:508-11.

8. Hamou J, Salat-Baroux J, Cupez F, De Brux J. Microhysteroscopy: a new approach to the diagnosis of cervical intraepithelial neoplasia. J Obstet Gynecol 1984; 63:567-74.

9. Andersen W, Frierson H, Barber S, Tabbarah S, Taylor P. Underwood P. Sensitivity and specificity of endocervical curettage and the endocervical brush for the evaluation of the endocervical canal. Am J Obstet Gynecol 1988; 159:702-7.

10.Saunders N, Anderson D, Gilbert L, Sharp F. Unsatisfactory colposcopy and the response to orally administered oestrogen: a randomized double blind placebo controlled trial. Br J Obstet Gynaecol 1990; 97:731-3.

11. Oyesanya OA, Amerasinghe C, Manning EA. A comparison between loop diathermy conization and coldknife conization for management of cervical dysplasia associated with unsatisfactory colposcopy. Gynecol Oncol 1993; 50:84-8.

12.Stern JL, Major C, Van Le L. Preventing cervical conization by achieving satisfactory colposcopy with hygroscopic cervical dilators. Am J Obstet Gynecol 1990; 163:176-7.

13.McCord ML, Stovall TG, Summitt RL Jr, Lipscomb GH, Collins KW, Parsons LH. Synthetic hygroscopic cervical dilator use in patients with unsatisfactory colposcopy. Obstet Gynecol 1995; 85:30-2.

14.Johnson N, Brady J. Dilating the cervix medically to overcome an unsatisfactory colposcopy: 5 year follow up. Eur J Obstet Gynecol Reprod Biol 1996; 69:125-7.

15.Borgatta L, Lopatinsky I, Shaw FM. Overcoming unsatisfactory colposcopy. Use of osmotic dilators. J Reprod Med 1997; 42:271-5.

16.Soutter WP, de Barros Lopes A, Fletcher A, et al. Invasive cervical cancer after conservative therapy for cervical intraepithelial neoplasia. Lancet 1997; 349:978-80.

17.Dietrich CS $3^{\text {rd }}$, Yancey MK, Miyazawa K, Williams DL, Farley J. Risk factors early cytologic abnormali- 
ties after loop electrosurgical excision procedure. Obstet Gynecol 2002; 99:188-92.

18. Costa S, De Nuzzo M, Terzano P, et al. Factors associated with cone margin involvement in CIN patients undergoing conization-equivalent electrosurgical procedure. Acta Obstet Gynecol Scand 2000; 79:586-92.

19.Jakus S, Edmonds P, Dunton C, King SA. Margin status and excision of cervical intraepithelial neoplasia: a review. Obstet Gynecol Surv 2000; 55:520-7.

20.Johnson N, Crompton AC. A new way to expose endocervical lesions at colposcopy. Gynecol Oncol 1990; 36:217-8.

Conflito de interesses: Os dilatadores utilizados neste trabalho foram fornecidos pelas empresas Merocel (Lamicel) e FEMA International (Dilapan-S).
21.Rieper JP. Colposcopia endocervical. Rev Ginecol Obstet 1965; 117:239-43.

22.Lopes A, Pearson SE, Mor-Yosef S, Ireland D, Monaghan JM. Is it time for a reconsideration of the criteria for cone biopsy ? Br J Obstet Gynaecol 1989; 96:1345-7.

23.Dôres GB. Cirurgia de alta freqüência no tratamento da neoplasia intra-epitelial grau III do colo uterino [tese]. São Paulo: Univ. Federal de São Paulo; 1996.

24.Cardoso MS, Ribalta JCL, Taha NS, Focchi J, Baracat EC, Lima GR. Resultados da cirurgia com alça de alta freqüência e localização colposcópica da área com atipia ectocervical. Rev Bras Ginecol Obstet $2001 ; 23: 349-54$.

25.Tseng CJ, Liang CC, Lin CT, et al. A study of diagnostic failure of loop conization in microinvasive carcinoma of the cervix. Gynecol Oncol 1999; 73:91-5.

Recebido em: 2/ 12/2003 Aceito com modificações em: 19/1/2004

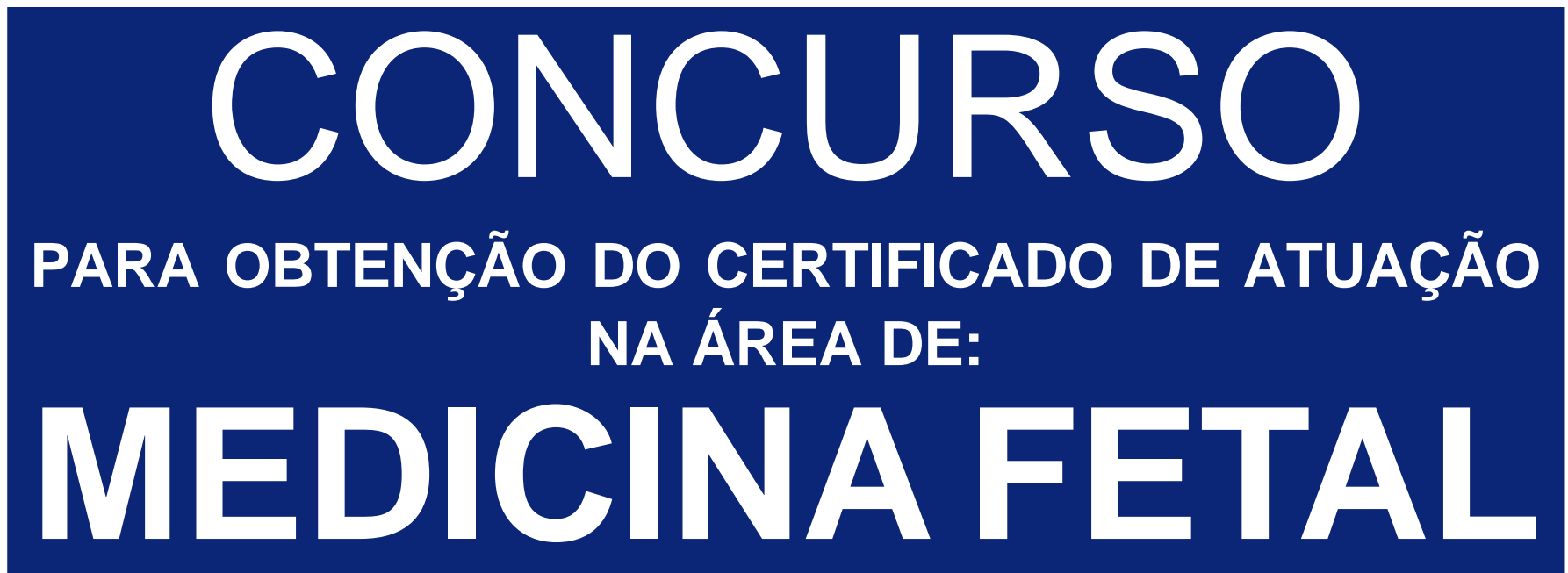

\title{
PERÍODO PARA ENVIO DO CURRÍCULUM: $15 / 04 / 2004$ A 14/06/2004
}

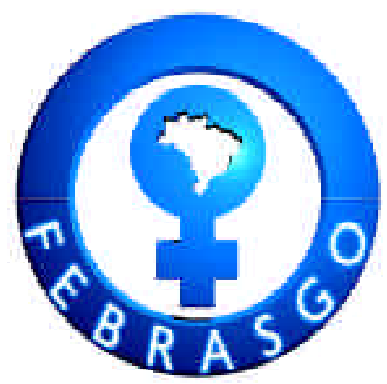

\author{
EDITAL E FICHA DE INSCRIÇÃO: \\ Retirar na Home Page da FEBRASGO \\ www.febrasgo.org.br
}

ENVIAR PARA SECRETARIA EXECUTIVA DA FEBRASGO:

Av. das Américas, 8445 - sala 711

Barra da Tijuca - Rio de Janeiro - RJ

22793-081

Tel.: (0xx21) 2487-6336 